\title{
On Higher Order Gravities, Their Analogy to GR, and Dimensional Dependent Version of Duff's Trace Anomaly Relation
}

\author{
Mehrdad Farhoudi* \\ Department of Physics, Shahid Beheshti University, \\ Evin, Tehran 1983963113, Iran
}

October 31, 2005

\begin{abstract}
An almost brief, though lengthy, review introduction about the long history of higher order gravities and their applications, as employed in the literature, is provided. We review the analogous procedure between higher order gravities and GR, as described in our previous works, in order to highlight its important achievements. Amongst which are presentation of an easy classification of higher order Lagrangians and its employment as a criteria in order to distinguish correct metric theories of gravity. For example, it does not permit the inclusion of only one of the second order Lagrangians in isolation. But, it does allow the inclusion of the cosmological term. We also discuss on the compatibility of our procedure and the Mach idea. We derive a dimensional dependent version of Duff's trace anomaly relation, which in four-dimension is the same as the usual Duff relation. The Lanczos Lagrangian satisfies this new constraint in any dimension. The square of the Weyl tensor identically satisfies it independent of dimension, however, this Lagrangian satisfies the previous relation only in three and four dimensions.
\end{abstract}

PACS number: 04.20.-q; 04.50.+h

Keywords: Higher order gravity; Non-linear Lagrangians; Weyl anomaly.

\section{Higher Order Gravity Review}

In spite of the success of Einstein's gravitational theory, it can only be considered as a step towards a much more complete and comprehensive structure due to a number of seeming weaknesses. Even its own past successes have also raised deep and difficult problems, e.g. it has not yet been

*m-farhoudi@sbu.ac.ir 
brought into a unified theory of nature [1]. The failure to establish a proper quantum theory of gravity presently poses basic questions concerning the scope and general formalism of such a theory itself, and induces a search for alternative theories of gravitation [2]. Interest in this subject stems mostly from making a systematic investigation into the foundations of general relativity amongst which are fundamental changes in dynamics and dimension of space-time.

The principle of general invariance imposes that the action integral for gravity must be an invariant quantity, indeed, the profound implications of this state of affairs are due to Hilbert [3] who was first to employ the necessity of the general invariance of all physical laws to the variational principle. Then, the use of the action principle and the principle of general invariance allow an immediate connection between symmetry principles and conservation laws to be established as inner identities. Yet, change of dynamics can mainly be achieved by altering Lagrangian of a system, as in general relativity (GR) the physical requirements which should be satisfied by any reasonable Lagrangian formulation of gravitational interactions are still an open subject for discussion.

Among scalar Lagrangians, field equations based on a Lagrangian quadratic in the curvature tensor have had a long history in the theory of gravitation. These theories occur for various reasons in different areas of theoretical physics. Though, they have taken much more attraction in the last two decades when they have been proposed mainly to solve some problems in the quantization of gravity. But, the first idea dates back to the early days of GR in the work by Weyl [4] and Eddington [5] in an attempt to unify gravity with electromagnetism, however this approach was unfruitful [6, 7].

Then, Bach [8] and Lanczos 9] considered actions that are made up only by the quadratic terms in the curvature in four dimensions as scaleinvariance, due to Weyl's idea of the principle of gauge-invariance under conformal transformations for when the conformal factor is a constant, e.g. they studied the dimensionless action $\int R^{2} \sqrt{-g} d^{4} x$. However, this idea has also been strongly criticized as a nonviable theory [10]. The two main objections against these Lagrangians are as the metric based on them does not satisfy the flat space limit at asymptotically large distances, and disagreement with observations follows when the matter is incorporated. A Lagrangian proportional to $R^{1+\delta}$ has also been investigated [11, and it has been shown that the observational constraints leads to the overall bound $0 \leq \delta<7.2 \times 10^{-19}$. Exact cosmological solutions of this type of scale-invariant gravity theories have been considered in [12].

Since these early suggestions, work in this area was not very active, although some results were obtained ${ }^{1}$ before it flourished in the seventies, i.e. after suggestions made from considering the quantization of GR in the

\footnotetext{
${ }^{1}$ See, for example, Ref. [13.
} 
sixties.

Perhaps a legitimate mathematical motivation to examine gravitational theories built on non-linear Lagrangians has been the phenomenological character of Einstein's theory which leaves room for such amendments, i.e. the dependence of the Einstein tensor/Lagrangian on the derivatives of the metric and the dimension. Actually, the Einstein Lagrangian is not the most general second order Lagrangian allowed by the principle of general invariance, and indeed, through this principle the latter generalization can be performed up to any order, and a general scalar Lagrangian is a higher derivative Lagrangian.

However, Einstein's gravitational theory has proven to be a successful theory in "real" physical phenomena, e.g. in the "weak" gravitational field, and its main difficulties become manifest only when the curvature of spacetime is not negligible. Actually, the curvature is noticeable on very small scales, and this is particularly relevant to the very early universe. In another words, at really small distances, of the order of Planck's length, an Euclidean topological structure is quite unlikely. At such distances, even the fluctuations of quantum gravitation will be extremely violent and probably produce an ever changing, dynamic topology [14. Thus, it perhaps allows Lagrangians extended beyond the Einstein-Hilbert Lagrangian to be considered as alternative theories.

On the other hand, the unity of physics during its development must be maintained by the correspondence principle. That is, in every "new" physical theory the "previous" one is contained as a "limiting" case. As mentioned before, gravitational theories based on a Lagrangian which is only purely quadratic in the curvature tensor have been strongly criticized 10. as nonviable theories. However, a gravitational theory has to not only correctly reflect the dynamical behavior of the whole universe, but also be valid for stellar evolution. Therefore, one should demand that GR must be maintained as a "limiting" case of non-linear gravitational theories.

Evidently, the unification programs in particle physics seem to demand energies of the order of cosmological energies, $E \geq 10^{15} \mathrm{GeV}$, in order to be verified, and thus the very early universe may provide the only place to test these ideas. Hence, the standard prejudices about the strength of quantum effects on gravitational interaction imply that they should not be appreciable unless the distances involved in the problem are very small, of the order of Planck's length. So, the questions of quantum gravity are naturally connected with the very early universe cosmology [15]. That is, these effects should be completely negligible in the "limiting" case of nonlinear gravitational Lagrangians, i.e. in the low energy regime when $E \ll$ $E_{p} \approx 10^{19} \mathrm{GeV}$.

It is believed that higher order Lagrangians play an essential rôle for 
supergravity approach [16. Nowadays, it is also well known ${ }^{2}$ that Einstein's gravity when treated as a fundamental quantum gravity leads to a non-renormalizable theory, although, these difficulties become manifest only when the curvature of space-time is not negligible. In order to permit renormalization of the divergences, quantum gravity in curved space-time has indicated that the Einstein-Hilbert action should be enlarged by the inclusion of higher order curvature counter terms 18. In fact, it has been shown $^{2}$ that the Lagrangian ${ }^{3}$

$$
L=\frac{1}{\kappa^{2}}\left(R+\alpha R^{2}+\beta R_{\mu \nu} R^{\mu \nu}\right)
$$

which has the required Newtonian limit and, by the Gauss-Bonnet theorem (relation) [19, is the most general quadratic Lagrangian in (and up to) four dimensions, solves the renormalization problem and is multiplicatively renormalizable [20] and asymptotically free [21]. However, it is not unitary within usual perturbation theory [6]. The analysis of quantum theory revealed [20] that the particle spectrum of the Lagrangian (10), in general, contains, besides the massless spin-two particles (i.e. gravitons), a further massive spin-two particle (i.e. ghost) and a massive scalar (spin-zero) particle, with a total of eight degrees of freedom. In the limit $\beta \rightarrow 0$, the spin-two ghost disappears, however, the divergence problems in the quantum theory reappear [6].

The inclusion of higher order Lagrangian terms have also appeared in the effect of string theory on classical gravitational physics by means of a low energy effective action which expresses gravity at the classical level [22, 23]. This effective action in general gives rise to fourth order field equations (and brings in ghosts), and in particular cases, i.e. in the form of dimensionally continued Gauss-Bonnet densities, it is exactly the Lovelock terms ${ }^{4}$ (and

\footnotetext{
${ }^{2}$ See, for example, Refs. [17] and references therein.

${ }^{3} \alpha, \beta$ and $\kappa^{2} \equiv \frac{16 \pi G}{c^{4}}$ are constants, and where field equations are shown as $G_{\alpha \beta}^{\text {(gravitation) }}=\frac{1}{2} \kappa^{2} T_{\alpha \beta}$.

${ }^{\alpha}$ The Lovelock Lagrangian, in a $D$ dimensional space-time, is [24] 25]

$$
\mathcal{L}=\frac{1}{\kappa^{2}} \sum_{0<n<\frac{D}{2}} \frac{1}{2^{n}} c_{n} \delta_{\beta_{1} \ldots \beta_{2 n}}^{\alpha_{1} \ldots \alpha_{2 n}} R_{\alpha_{1} \alpha_{2}}^{\beta_{1} \beta_{2}} \cdots R_{\alpha_{2 n-1} \alpha_{2 n}}^{\beta_{2 n-1} \beta_{2 n}} \equiv \sum_{0<n<\frac{D}{2}} c_{n} L^{(n)}
$$
}

where we set $c_{1} \equiv 1$ and the other $c_{n}$ constants to be of the order of Planck's length to the power $2(n-1)$, for the dimension of $\mathcal{L}$ to be the same as $L^{(1)} \equiv L_{E-H}=R$. $\delta_{\beta_{1} \ldots \beta_{p}}^{\alpha_{1} \ldots \alpha_{p}}$ is the generalized Kronecker delta symbol, which is identically zero if $p>D$ and the maximum value of $n$ is related to the dimension of space-time by

$$
n_{\max }= \begin{cases}\frac{D}{2}-1 & \text { even } D \\ \frac{D-1}{2} & \text { odd } D\end{cases}
$$

Hence, $\mathcal{L}$ reduces to the Einstein-Hilbert Lagrangian in four dimensions. Implicitly, we follow the sign conventions of Wald [26]. 
consequently no ghosts arise) 27]. However, Duff et al 28] claimed that the quadratic contribution to the low energy effective Lagrangians for the closed bosonic string is of the form $R^{2}-4 R_{\mu \nu} R^{\mu \nu}+2 R_{\alpha \beta \mu \nu} R^{\alpha \beta \mu \nu}$, for the Type II is of the form $R^{2}-4 R_{\mu \nu} R^{\mu \nu}$ and only for the $E_{8} \times E_{8}$ heterotic string do form the Gauss-Bonnet combination. The latter term appears naturally in the next-to-leading order term of the heterotic string effective action and also plays an essential role in the Chern-Simons gravitational theories [29, 30].

Incidentally, recent argument in 31. shows that $\frac{1}{R}$ modification term also follows from certain compactification schemes of string/M-theory.

Though, higher derivative terms are often added as a correction to the ordinary, lower derivative, theory of gravity, but these terms do not only mean that they will perturb the original theory. Actually, their presence, as unconstrained terms even with small coefficients, make the new theory completely different from the original one 32 .

Besides, there has been much attraction in considering gravity in higher dimensional space-time. In this context, one may also use a consistent theory of gravity with a more general action, e.g. the Einstein-Hilbert action plus higher order terms. Indeed, the above ghost-free property, and the fact that the Lovelock Lagrangian is the most general second order Lagrangian which, the same as the Einstein Lagrangian, yields the field equations as second order equations, have stimulated interests in the Lovelock gravity and its applications. A considerable amount of work has been done in this area, especially in the eighties, and perhaps, the greatest number of applications are performed in cosmology $33,,^{5}$ amongst which are approaches to inflationary scenarios. Recent attraction engages it with the brane cosmology and the cosmic acceleration [38, 39].

Actually, the observation of high red-shift supernova [40] and the measurement of angular fluctuations of cosmic microwave background fluctuations [41] have separately, though the former directly and the latter indirectly, established that the universe expands with acceleration, instead of deceleration, at the present epoch. Henceforth, as an alternative approach to the vacuum energy (the cosmological constant) and/or additional scalar field (quintessence), modifications of gravity itself with different terms of higher order gravity have recently come into consideration [39], 42 - 48, for, e.g., the $\frac{1}{R}$ modified term grows when curvature decreases and it may produce the cosmic acceleration. However, Ref. [46] claims that they have found a linearly growing force which is not phenomenologically acceptable. Besides, the $\frac{1}{R}$ term modification has been shown to lead to instabilities [45]- 47]. Though, Ref. 44] claims that further modification of this modified gravity by $R^{2}$ or other higher order terms may resolve the instabilities, or perhaps

\footnotetext{
${ }^{5}$ For a more recent work on this issue see, e.g. Ref. [34.

${ }^{6}$ See, for example, Refs. 35]-37. and references therein.
} 
make them avoidable [43, 48].

The charges associated to the diffeomorphism symmetries of Lovelock gravity in any odd dimensional space-time has been investigated in 30. The boundary term [4], Lovelock-Cartan gravity [50] and Palatini's device 51] for non-linear Lagrangians have been considered as well. Though, Buchdahl [52] has claimed the mutual in-equivalence of $\mathrm{H}$-variation and $\mathrm{P}$ variation, and has illustrated this for quadratic Lagrangians as an example. However, it has been shown [53] that the latter Lagrangian still satisfies Birkhoff's theorem. For recent work on this issue, see e.g. Refs. 54. Besides, it has been claimed [55] that the Palatini formalism of the $\frac{1}{R}$ modified gravity has no such mentioned instabilities, but still provides the current observations. Actually, Ref. [56] claims that the Palatini formalism can provide a mechanism to explain the cosmic acceleration without the necessity of dark energy sources. However, new instabilities from quantum effects may appear [57]. But, Ref. 48] shows these new instabilities may be suppressed by quantum effects of conformal fields, and Ref. [58 argues that the conclusion for the new instabilities is false, for mathematical equivalence, and not physical equivalence, has been used.

Non-linear Lagrangians are also proposed in the context of relativistic cosmology in order to avoid the appearance of some of the singularities typically encountered for solutions of GR? The general behavior of cosmology, the existence and stability of special solutions, like de Sitter, have been considered for theories with $f(R)$ Lagrangian in, e.g., Refs. 60]. For recent work on this issue see Ref. 61] and references therein.

Because of the higher order and greater degree of the non-linearity of the field equations, it is very difficult to find out non-trivial exact analytical solutions of Einstein's equation with higher order terms, and hence, extract physical predictions from them, especially that the present technology hardly may provide empirical checks on these predictions. Though, the Newtonian limit of fourth [6, 62] and higher 63] order gravities are described by a Newtonian plus Yukawa term potential. Recently, the post-Newtonain parameters limit of such theories, especially $f(R)$ Lagrangian with corrections that grow at low curvatures, has been analyzed both in the metric and in the Palatini formalisms [45, 46, 64, 65] with contrasting results for being compatible with solar system observations. As, e.g., Ref. 65] claims that the analyzes performed in some works are not founded since they are obtained at the low energy approximation, which is not the case inside the entire solar system. For a more recent work on this issue see Ref. 66.

The Cauchy problem for the fourth order field equations deriving from $R+\alpha R^{2}$ is essentially reducible to a second order one [67] and its energy is a positive definite quantity 68. The positive energy theorem has also been shown to hold for a larger class of non-linear Lagrangians for which

\footnotetext{
${ }^{7}$ See, for example, Refs. [59].
} 
the Einstein frame can be defined around flat space [69].

In general, the reduction of non-linear purely metric Lagrangian theories of gravity to a second order form, as equivalent theories to GR plus additional matter fields, so-called "dynamical universality" of Einstein's gravity, under conformal/Legendre like transformations, has been discussed in the literature, see, e.g., Refs. [70] and references therein. Although, it has raised a question on the real physical metric [71, where, in reply [72, comments are in having the dynamical equivalence of non-singular conformal transformations where then, the systems are isomorphic, and not having necessarily the physical equivalence. Also, the dynamical equivalence of arbitrary high order extended theories of gravity with scalar-tensor gravity, which claims to be conformally equivalent to GR plus scalar fields, has been considered 62, 74, 75. More discussion about physical equivalence of nonlinear gravity theories, based on the physical features of energy, has been presented in Ref. 69.

Some static black hole solutions of the Gauss-Bonnet gravity have been obtained in [6]. And equations governing relativistic fluid dynamics for quadratic theories and $f(R)$ theories of gravity have also been worked out [7].

Finally, considering the corresponding Euler-Lagrange expressions of Lagrangians containing the derivatives of the curvature scalar are firstly due to Buchdahl [78. In general, $f\left(\sum_{k=0}^{p} \square^{k} R\right)$ Lagrangian, where $p$ is a positive integer and among which is the well known sixth order gravity [35, 37, 75, 78, and its generalization [79, has been considered in the literature as well. The corresponding Euler-Lagrange expression for the special case of $f(R)$ has been given in [80], and the power series example, i.e. $f(R)=\sum_{k=0}^{p} a_{k} R^{k}$, has also been used in 81 .

Yet, very vast amount of work has been done in the higher order gravities and the references given in this compact survey are not obviously a complete bibliography on this issue. Actually, this section is almost brief, though lengthy, review introduction about the long history of higher order gravities and their applications. In the next section, we will introduce what we have achieved on this subject.

\section{Introduction}

Getting interested in higher order gravities, we have noticed that the mathematical form of the Einstein tensor, i.e. the splitting feature of it into the Ricci tensor and the curvature scalar with the trace relation between them is also a common remarkable properties of each homogeneous term

\footnotetext{
${ }^{8}$ Also, see Ref. 73 .
} 
in the Lovelock tensor, 9 but not true for the whole Lovelock tensor. In our previous work [82, we demanded an analogy that this common feature should also be valid for the Lovelock tensor itself, i.e. $\mathcal{G}_{\alpha \beta}=\Re_{\alpha \beta}-\frac{1}{2} g_{\alpha \beta} \Re$ with $\operatorname{Trace} \Re_{\alpha \beta}=\Re$, or generally, for any inhomogeneous Euler-Lagrange expression constructed linearly in terms of homogeneous terms. Indeed, we did this via defining a generalized trace operator, that we denoted by Trace. ${ }^{10}$

Then, we took 83] the above analogy further for the alternative form of the Lovelock equation to be the same as the appearance of the alternative form of the Einstein equation. Hence, we found that the price for this analogy is to accept the existence of the trace anomaly of the energy-momentum tensor even in classical treatments. That is, we have actually stated a classical view of gravitation which explicitly shows the presence of an extra (anomalous) trace for the energy-momentum tensor, with an indication of the constitution of the higher order gravities towards this trace anomaly, exactly as what has been verified in the quantum aspects of gravity [84, 85].

As an example, we employed [83] this analogy to any generic, the most general, second order Lagrangian

$$
L_{\text {generic }}^{(2)}=\frac{1}{\kappa^{2}}\left(a_{1} R^{2}+a_{2} R_{\mu \nu} R^{\mu \nu}+a_{3} R_{\alpha \beta \mu \nu} R^{\alpha \beta \mu \nu}\right),
$$

\footnotetext{
${ }^{9}$ That is $G_{\alpha \beta}^{(n)}$, where the Lovelock tensor, as dimensionally reduction Euler-Lagrange terms in a $D$ dimensional space-time, is [24, 25]

$$
\mathcal{G}_{\alpha \beta}=-\sum_{0<n<\frac{D}{2}} \frac{1}{2^{n+1}} c_{n} g_{\alpha \mu} \delta_{\beta \beta_{1} \ldots \beta_{2 n}}^{\mu \alpha_{1} \ldots \alpha_{2 n}} R_{\alpha_{1} \alpha_{2}}^{\beta_{1} \beta_{2}} \cdots R_{\alpha_{2 n-1} \alpha_{2 n}}^{\beta_{2 n-1} \beta_{2 n}} \equiv \sum_{0<n<\frac{D}{2}} c_{n} G_{\alpha \beta}^{(n)}
$$
}

where the cosmological term has been neglected and $G_{\alpha \beta}^{(1)} \equiv G_{\alpha \beta}$, i.e. the Einstein tensor.

${ }^{10}$ That is, for a general $\left(\begin{array}{l}N \\ M\end{array}\right)$ tensor which is a homogeneous function of degree $h$ with respect to the metric and its derivatives, we defined

$$
\operatorname{Trace}^{[h]} A^{\alpha_{1} \ldots \alpha_{N}}{ }_{\beta_{1} \ldots \beta_{M}}:= \begin{cases}\frac{1}{h-\frac{N}{2}+\frac{M}{2}} \operatorname{trace}^{[h]} A^{\alpha_{1} \ldots \alpha_{N}}{ }_{\beta_{1} \ldots \beta_{M}} & \text { when } h-\frac{N}{2}+\frac{M}{2} \neq 0 \\ \operatorname{trace}^{[h]} A^{\alpha_{1} \ldots \alpha_{N}} \beta_{1} \ldots \beta_{M} & \text { when } h-\frac{N}{2}+\frac{M}{2}=0 .\end{cases}
$$

Hence, for example, when $h \neq 0$ and $h^{\prime} \neq 0$, one gets

$$
\text { Trace }\left({ }^{\left[h^{\prime}\right]} C^{[h]} A_{\mu \nu}\right)= \begin{cases}\frac{h+1}{h^{\prime}+h+1}{ }^{\left[h^{\prime}\right]} C \operatorname{Trace}^{[h]} A_{\mu \nu} & \text { for } h \neq-1 \\ \frac{1}{h^{\prime}}{ }^{\left[h^{\prime}\right]} C \operatorname{Trace}^{[h]} A_{\mu \nu} & \text { for } h=-1 .\end{cases}
$$

As mentioned, the homogeneity is taken with respect to the metric and its derivatives, without loss of generality, with the homogeneity degree number (HDN) conventions of ${ }^{[+1]} g^{\mu \nu}$ and ${ }^{[+1]} g^{\mu \nu}{ }_{, \alpha}$. Hence, one can relate the orders $n$ in any Lagrangian, as in $L^{(n)}$, that represents its HDN. See Ref. 82 for details. 
in $D \geq 3$ dimension. ${ }^{11}$ Its corresponding Euler-Lagrange expression is

$$
\begin{aligned}
& G_{\text {(generic) } \alpha \beta}^{(2)} \equiv \frac{\kappa^{2}}{\sqrt{-g}} \frac{\delta\left(L_{\text {generic }}^{(2)} \sqrt{-g}\right)}{\delta g^{\alpha \beta}} \\
& =2\left[a_{1} R R_{\alpha \beta}-2 a_{3} R_{\alpha \mu} R_{\beta}{ }^{\mu}+a_{3} R_{\alpha \rho \mu \nu} R_{\beta}{ }^{\rho \mu \nu}+\left(a_{2}+2 a_{3}\right) R_{\alpha \mu \beta \nu} R^{\mu \nu}\right. \\
& \left.\quad-\left(a_{1}+\frac{1}{2} a_{2}+a_{3}\right) R_{; \alpha \beta}+\left(\frac{1}{2} a_{2}+2 a_{3}\right) \square R_{\alpha \beta}\right] \\
& \quad-\frac{1}{2} g_{\alpha \beta}\left[\kappa^{2} L_{\text {generic }}^{(2)}-\left(4 a_{1}+a_{2}\right) \square R\right],
\end{aligned}
$$

where $\square \equiv ; \rho^{\rho}$, and where it can be written as

$$
G_{(\text {generic }) \alpha \beta}^{(2)} \equiv R_{(\text {generic }) \alpha \beta}^{(2)}-\frac{1}{2} g_{\alpha \beta} R_{\text {generic }}^{(2)} .
$$

Then, in order that the "trace" relation, Trace $R_{(\text {generic }) \alpha \beta}^{(2)}=R_{\text {generic }}^{(2)}$, to be satisfied, we exactly derived the trace anomaly relation

$$
3 a_{1}+a_{2}+a_{3}=0 .
$$

which, in the process of re-examining the Weyl anomaly's applications, was also suggested by Duff [86] in any dimension.

Note that, as a homogeneous Euler-Lagrange expression has a uniform HDN, then one can work with the usual trace instead of the Trace operator, and obtains the same results if one demands the appropriate "trace" relation, i.e. $\frac{1}{n} \operatorname{trace} R_{(\text {generic) } \alpha \beta}^{(n)}=R_{\text {generic }}^{(n)}$. Though, the notion of Trace operator was introduced as to be able to deal with when one considers the Einstein-Hilbert Lagrangian plus higher order terms as a complete gravitational Lagrangian, i.e. when one works with an inhomogeneous Lagrangian constructed linearly in terms of homogeneous terms.

One may conclude that the appearance of trace anomaly maybe interpreted as Lovelock modification of gravity even in classical treatments. Though, the above consistency condition has also been derived [87, 88, based on a cohomological point of view, using the Wess-Zumino consistency conditions, which was claimed to be the true reason for the existence of such a relation. It has also been recently claimed that the AdS/CFT correspondence, namely the holographic conformal anomaly, maybe responsible for it, see e.g. Ref. [89] and references therein.

The extra trace of the energy-momentum tensor for generic cases is 83

$$
T^{\prime}=-\kappa^{-2} D \sum_{n \geq 1} \frac{n-1}{n} c_{n} R_{\text {generic }}^{(n)} \equiv \sum_{n \geq 1} T_{n}^{\prime},
$$

\footnotetext{
${ }^{11}$ The $a_{1}, a_{2}$ and $a_{3}$ are arbitrary dimensionless constants, and obviously, in three and four dimensions, only two of these three terms are effective. And in two dimensions, only one term survives.
} 
where $R_{\text {generic }}^{(1)} \equiv R$ (however, $T_{1}^{\prime}=0$ ), and there is no upper limit for $n$ in generic cases.

For the above example of $n=2$, it is

$$
T_{2}^{\prime}=-\frac{\kappa^{-2} D}{2} c_{2} R_{\text {generic }}^{(2)},
$$

where $R_{\text {generic }}^{(2)}$, according to equations (8) and (9), is

$$
R_{\text {generic }}^{(2)} \equiv \kappa^{2} L_{\text {generic }}^{(2)}-\left(4 a_{1}+a_{2}\right) \square R,
$$

with constraint (10). The relation (12) is exactly the same as the relevant most general form of the anomalous trace of the energy-momentum tensor for classically conformally invariant fields of arbitrary spin and dimension which has been shown 90, to be

$$
\left\langle T_{\rho}{ }^{\rho}\right\rangle_{\mathrm{ren}}=-\frac{\hbar c}{180(4 \pi)^{2}}\left(a_{1} R^{2}+a_{2} R_{\mu \nu} R^{\mu \nu}+a_{3} R_{\alpha \beta \mu \nu} R^{\alpha \beta \mu \nu}+\gamma \square R\right) .
$$

This obviously shows that

$$
\gamma=-\left(4 a_{1}+a_{2}\right)
$$

which it completes the trace anomaly relation suggested by Duff [86]. However, the calculations by most authors confirmed [86] the constraint (10) for the values of $a_{1}, a_{2}$ and $a_{3}$ in all cases, but not always that of $\gamma$. Apparently, this is due to the fact that the term $\square R$ is a local anomaly [86]. The ambiguities of the four dimensional anomaly has been recently investigated in Ref. 85.

Actually, in the semi-classical approach of quantum gravity theory employed to deduce the trace anomalies, the effective action is 91] a covariant functional i.e., invariant under diffeomorphisms and local gauge transformations. Therefore, the approximation procedures for calculating the effective action have to preserve the general covariance at each order. Hence, conformal invariance 92 is also sacrificed to the needs of general covariance, i.e. the Weyl conformal invariance is not a good symmetry beyond the classical level. This is what we have also performed in the classical theory of gravitation through preserving the covariant property of the linear Lagrangian theory of Einstein's gravity for each order of non-linear theories of gravitation, by an analogous demand.

Hence, the origin of Duff's suggested relation between the coefficients of the conformal anomalies may classically be interpreted due to the general covariance of Einstein's theory as applied to the second order of the Lovelock modification of gravity. Though, it is somehow a naive conjecture, nevertheless, it gives almost an easy classical procedure to grasp the desired result, which, (perhaps) besides what have been already given in the literature [87, 88, 89], may indicate of an intrinsic property behind it. 
In this article, first in the following section, in order to present a better view of the analogy/approach that we have employed, we will summarize its important achievements, which mainly have not been highlighted before. Then, in Section 4, based on this analogy, we will derive a dimensional dependent version of Duff's suggested relation for trace anomaly.

\section{Remarks}

In this section, we will outline what mainly have been gained in the above mentioned analogous procedure till now, which have not been emphasized or stated in our previous works. Aimed to show, as a main advantage, that this analogous of the Einstein tensor can be employed as a criteria in order to distinguish correct/legitimate metric theories of gravity, which are either homogeneous functions or linear combinations of different homogeneous functions of the metric and its derivatives.

These remarks are as follows:

- If one allows scalar Lagrangian to be up to the $k^{\text {th }}$ order jet-prolongation of the metric, with $k \geq 2$, there would be a lot of Lagrangian choices that can be considered. For example, higher-loop quantum corrections to GR are expected to contain terms of the type $R \square^{k} R$ in the Lagrangian [23, 75]. That is, the gravitational theory with the Lagrangian

$$
L=\frac{1}{\kappa^{2}}\left(R+\sum_{k=0}^{p} a_{k} R \square^{k} R\right),
$$

where $p$ is a positive integer and $a_{k}$ 's are constants of the order of (Planck's) length to the power $2 k+2$. The above Lagrangian is up to $(2 p+2)^{t h}$ order jet-prolongation of the metric which, in general, leads to the gravitational field equations of order $2 p+4$. For $p=1$, the Lagrangian is

$$
L=\frac{1}{\kappa^{2}}\left(R+a_{0} R^{2}+a_{1} R \square R\right),
$$

which is known [75] as sixth order gravity in the literature, i.e. it leads to sixth order field equations, and wherein one should set $a_{0} \geq 0$ and $a_{1}<0$ in order to exclude tachyons [37.

Now, a point is that: the HDN provides an easy procedure to classify different Lagrangian terms, especially higher order Lagrangians.

For example, in order to amend the Lagrangian of sixth order gravity, Berkin et al 35] discussed that the Lagrangian term of $R \square R$ is a third order Lagrangian based on the dimensionality scale, i.e. two 
derivatives are dimensionally equivalent to one Riemann-Christoffel tensor or any one of its contractions. However, it can be better justified on account of the above regard, since it has the HDN three 82. Hence, in order to generalize Lagrangian (17), the following Lagrangian has also been considered in the literature [35, 37]

$$
L=\frac{1}{\kappa^{2}}\left(R+a_{0} R^{2}+b R^{3}+a_{1} R \square R\right)
$$

where $b$ is a constant of the order of (Planck's) length to the power 4 . Later on, the Lagrangian

$$
L=\frac{1}{\kappa^{2}}\left[R+a_{0} R^{2}+a_{N}(-R)^{N} \square R\right],
$$

where $N$ is a positive integer and $a_{N}$ is a constant of the order of (Planck's) length to the power $2 N+2$, has been regarded as generalized sixth order gravity [79] $!^{12}$ Obviously, the HDN of $(-R)^{N} \square R$ is $N+$ 2 [82, and hence, Lagrangian (19) does not contain all the terms constructed from the curvature scalar with equal HDNs, e.g. see below.

As another example, in addition to the eight linearly independent terms which appear in the third order of the Lovelock Lagrangian and are up to the $2^{\text {nd }}$ order jet-prolongation of the metric with the HDN three, namely 93, 94, 95]

$$
\begin{array}{lllll}
R^{3} & R R_{\mu \nu} R^{\mu \nu} & R R_{\rho \tau \mu \nu} R^{\rho \tau \mu \nu} & R^{\mu \nu} R_{\mu \gamma} R_{\nu}^{\gamma} & R_{\rho \tau} R_{\mu \nu} R^{\rho \mu \tau \nu} \\
R_{\lambda \rho} R^{\lambda \tau \mu \nu} R^{\rho}{ }_{\tau \mu \nu} & R^{\sigma \tau}{ }_{\mu \nu} R^{\mu \nu}{ }_{\lambda \rho} R^{\lambda \rho}{ }_{\sigma \tau} & R^{\sigma \tau}{ }_{\mu \nu} R_{\sigma \rho}^{\mu \lambda} R_{\tau \lambda}^{\nu \rho}{ }_{\tau \lambda}, \quad(20)
\end{array}
$$

there are generally another nine ${ }^{13}$ linearly independent scalar terms constructed from the Riemann-Christoffel tensor and its contractions which can have the HDN of three as well, though they are up to $3^{\text {rd }}$ or even higher order jet-prolongation of the metric, namely

$$
\begin{array}{llll}
R \square R & R_{\mu \nu} \square R^{\mu \nu} & R_{\mu \nu \rho \tau} \square R^{\mu \nu \rho \tau} & R_{\mu \nu} R^{; \mu \nu} \quad R_{\mu \nu ; \rho} R^{\mu \rho ; \nu} \\
\square^{2} R & R_{; \rho} R^{; \rho} & R_{\mu \nu ; \rho} R^{\mu \nu ; \rho} & R_{\mu \nu \rho \tau ; \alpha} R^{\mu \nu \rho \tau ; \alpha} .
\end{array}
$$

The higher order terms become increasingly complex, e.g. the full expression for the fourth order of the Lovelock Lagrangian which are up to the $2^{\text {nd }}$ order jet-prolongation of the metric with the HDN four has 25 terms [25, 95, 97.

\footnotetext{
${ }^{12}$ The sign convention is such that the de Sitter space-time has a negative curvature value 79 .

${ }^{13}$ However, $\square^{2} R$ is a complete divergence and has no effect in the variation of the action. Besides, not all of their corresponding Euler-Lagrange expressions are independent [96], e.g. $R_{; \rho} R^{; \rho}$, upon integration covariantly by parts, can be transferred to a boundary term plus the $R \square R$ term.
} 
- The second term of the Lovelock Lagrangian, the Gauss-Bonnet term, does indeed satisfy the condition (10). In another words, the coefficients used in the Lanczos Lagrangian are specific to a equivalence class of any generic second order Lagrangians accepting our analogous demand.

- If the constraint (10) is applied to the Lagrangian made from the square of the Weyl conformal tensor, namely

$$
C_{\alpha \beta \mu \nu} C^{\alpha \beta \mu \nu}=\frac{2}{(D-1)(D-2)} R^{2}-\frac{4}{(D-2)} R_{\mu \nu} R^{\mu \nu}+R_{\alpha \beta \mu \nu} R^{\alpha \beta \mu \nu},
$$

gives

$$
3 a_{1}+a_{2}+a_{3}=\frac{6}{(D-1)(D-2)}-\frac{4}{(D-2)}+1=\frac{(D-3)(D-4)}{(D-1)(D-2)},
$$

which can only be zero when the dimension of space-time is either three or four. Though, in three dimension, the Weyl tensor is itself identically zero. And, as the action constructed by the square of the Weyl tensor, i.e. $I \equiv \int C_{\alpha \beta \mu \nu} C^{\alpha \beta \mu \nu} \sqrt{-g} d^{D} x$, conformally transforms as $I \rightarrow \Omega^{D-4} I$, it is thus conformal invariant only in four dimensions. ${ }^{14}$

Hence, following the classification of Ref. 98, the constraint (10) indicates that in four dimensions, the anomaly can have two contributions, a type $A$ anomaly, i.e. the Euler density invariant, and a type $B$ anomaly built from conformal invariants, i.e. the Weyl squared in four dimensions.

- The condition (10) does not permit the inclusion of only one of the second order Lagrangians in isolation, as mentioned before, difficulties also exist in quantum gravity when only one of these second order terms is considered [6, 20].

- As the most general quadratic Lagrangian in (and up to) four dimensions is (11), the condition (10) reads

$$
3 \alpha+\beta=0
$$

where $\alpha$ and $\beta$ are arbitrary non-zero constants.

In this case, the particle spectrum in the quantum theory does not contain the scalar particle, though this does not help the unitarity problems [6].

\footnotetext{
${ }^{14}$ Actually, in four dimensions the only local geometrical conformal invariant that can be constructed from the metric tensor and its first and second derivatives is $C_{\alpha \beta \mu \nu} C^{\alpha \beta \mu \nu} \sqrt{-g}$.
} 
- In two dimensions, as $R_{\alpha \beta \mu \nu} R^{\alpha \beta \mu \nu}=2 R_{\mu \nu} R^{\mu \nu}=R^{2}$, there is only one independent second order Lagrangian that can be considered as a higher derivative term [99]. However, condition (10) does not allow it, just as one cannot have Einstein's Lagrangian term in two dimensional space-time either and other options have been sought [100].

- To enquire about the cosmological term, let us investigate the analogy for this as well. Consistent with the aspect of equations (2) and (4) for the $n=0$ case (zero HDN), the Lagrangian $c_{0} L^{(0)} \equiv 2 \Lambda / \kappa^{2}$, a constant, produces the cosmological term, $G_{\mu \nu}^{(0)}=-\Lambda g_{\mu \nu}$, in the field equations. Hence, the exception value of the HDN in our definition of generalized trace, ${ }^{15}$ equation (5), maybe related to the cosmological term difficulty. Nevertheless, with our choice of definition for the generalized trace, we have Trace ${ }^{[+1]} g^{\mu \nu}=$ trace $g^{\mu \nu}=D$ and Trace ${ }^{[-1]} g_{\mu \nu}=$ trace $g_{\mu \nu}=D$, as though the dimension of space-time has not been altered.

Now, if $D \neq 2$, one may, for example, set

$$
G_{\mu \nu}^{(0)}=\left[\frac{2 \Lambda}{D-2} g_{\mu \nu}\right]-\frac{1}{2} g_{\mu \nu}\left[\frac{2 D \Lambda}{D-2}\right] \equiv R_{\mu \nu}^{(0)}-\frac{1}{2} g_{\mu \nu} R^{(0)},
$$

which holds in the trace relation, i.e. $\operatorname{Trace} R_{\mu \nu}^{(0)}=R^{(0)}$. Therefore, the inclusion of the cosmological term is allowed by our analogous demand.

- As final remark, we claim that the analogous demand, that we have introduced, provides a more compatible understanding with the Mach idea. Let us first elaborate a few words on the background of this issue.

Stachel [101] has pointed out that what went wrong in the last conclusion of the so-called Einstein's "hole" argument was that the point events of the space-time manifold had been incorrectly thought of as individuated independently of the field itself. That is, it is impossible to drag the metric field away from a physical point in empty spacetime and leave that physical point behind. Actually, Einstein himself realized [102] this as he wrote to Besso that nothing is physically real but the totality of space-time point coincidences. Later on, in an addendum [103. he placed great stress on the inseparability of the metric and the manifold.

Besides, according to the Aristotelian view [104, space is a plenum, i.e. it is inseparably associated with the material substance, and not a void. Hence, the properties of space are not independent of the material

\footnotetext{
${ }^{15}$ That is, e.g. for any tensor $T_{\mu \nu}$ with $\mathrm{HDN}$ of $h$, we set Trace ${ }^{[h]} T_{\mu \nu}=\frac{1}{h+1} \operatorname{trace} T_{\mu \nu}$ when $h \neq-1$, see Ref. 82 for details.
} 
bodies that move in it. Just as it is well known that, even though in Newtonian physics, space is a pre-existing stage on which material particles are the characters acting out the drama of physical events, but, in relativistic gravitational physics, space cannot be considered apart from the matter that is in it. And, as the mathematician E. Whittaker points out, the characters create the stage as they walk about on it 104. Actually, as it has been pointed out [105], a basic problem of Newtonian mechanics is that the extrinsic state of a point particle, i. e. its appearance in space and time (usually characterized by its position and velocity), is a priori independent of its intrinsic state (usually characterized by its mass). Hence, one may conclude that the properties of space-time in gravitational theories must be inseparable from the matter that is in it.

On the other hand, though the main critique of Mach and Einstein to absolute space, that it acts on everything but is not affected by anything, is not applied to GR, but, actually, according to the Mach idea, the question would be whether matter just modifies an already existing space-time structure, or whether it is the only source for its structure [106]. The former, a weaker version of Mach's principle, is in agreement with Einstein's gravitational theory. But, the latter, a strong version of Mach's principle in the sense that for a universe devoid of matter there should be no meaning for the existence of space time [106], is not consistent with it, for it has specified structures in the absence of matter, ${ }^{16}$ e.g. the Minkowski, Schwarzschild and TaubNUT metrics [108]. So that, the space-time of GR still itself has some essence independent of matter.

The link to this issue, in our opinion, could be the relation between $T$ and $R$ as in Trace $G_{\alpha \beta}=\left(1-\frac{D}{2}\right) R=\frac{1}{2} \kappa^{2} T$, and the basic concept of matter. For example, a similar behavior, as $R$ vanishes whenever $T_{\alpha \beta}$ vanishes, should also be sought for the higher order terms. This is somehow a procedure that it may indicate a more compatibility between GR and the strong version of the Mach idea [109. In another words, we adopt the view for establishing the generality of the known proposals, namely, that geometrical curvature induces matter [110, geometrical description of physical forces, and geometrical origin for the matter content of the universe. As the idea, more or less in this connection, that associates extra dimensions with the intrinsic characteristics of matter, e.g. 105], or any analytical $D$ dimensional Riemannian manifold can be locally embedded in an $(D+1)$ dimensional flat Riemannian manifold [111, see Refs. 112] for a more extensive discussion.

\footnotetext{
${ }^{16}$ See, for example Ref. [107].
} 
Hence, using the analogous demand, we showed 83] that in the cases where higher order gravities dominate, space-time "behaves" as if its energy-momentum has been "exchanged" with matter's energymomentum in the sense that in a universe devoid of "matter" there should be also no meaning for the existence of space-time. Note that, the applicability of higher order gravitational theories are restricted by the energy scale. In other words, as the coefficients of higher order gravities are very small, one cannot detect such implications in "real" world. However, these effects are important in highly curved areas, such as the very early universe, or in quantum physics.

\section{Dimensional Dependent Version of Duff's Rela- tion}

The appearance of $G_{(\text {generic } \alpha \beta}^{(2)}$ and the order through which one can define $R_{\text {(generic }) \alpha \beta}^{(2)}$ and $R_{\text {generic }}^{(2)}$ is somehow a critical point. In this section, we order/factorize the equation (8) in an alternative and more basic approach, which is commonly employed in the process of varying the action [82, namely

$$
\begin{aligned}
& G_{\text {(generic) } \alpha \beta}^{(2)} \equiv \frac{\kappa^{2}}{\sqrt{-g}} \frac{\delta\left(L_{\text {generic }}^{(2)} \sqrt{-g}\right)}{\delta g^{\alpha \beta}}=\kappa^{2} \frac{\delta\left(L_{\text {generic }}^{(2)}\right)}{\delta g^{\alpha \beta}}-\frac{1}{2} \kappa^{2} g_{\alpha \beta} L_{\text {generic }}^{(2)} \\
& =2\left[a_{1} R R_{\alpha \beta}-2 a_{3} R_{\alpha \mu} R_{\beta}^{\mu}+a_{3} R_{\alpha \rho \mu \nu} R_{\beta} \rho \mu \nu+\left(a_{2}+2 a_{3}\right) R_{\alpha \mu \beta \nu} R^{\mu \nu}\right. \\
& \quad-\left(a_{1}+\frac{1}{2} a_{2}+a_{3}\right) R_{; \alpha \beta}+\left(\frac{1}{2} a_{2}+2 a_{3}\right) \square R_{\alpha \beta}+\frac{1}{4}\left(4 a_{1}+a_{2}\right) \times \\
& \left.\quad g_{\alpha \beta} \square R\right]-\frac{1}{2} \kappa^{2} g_{\alpha \beta} L_{\text {generic }}^{(2)} \\
& \equiv R_{\text {(generic) } \alpha \beta}^{\left(2^{\prime}\right)}-\frac{1}{2} g_{\alpha \beta} R_{\text {generic }}^{\left(2^{\prime}\right)} .
\end{aligned}
$$

As it is evident, in this case, $R_{(\text {generic }) \alpha \beta}^{\left(2^{\prime}\right)} \equiv \kappa^{2} \frac{\delta\left(L_{\text {generic }}^{(2)}\right)}{\delta g^{\alpha \beta}}$ and $R_{\text {generic }}^{\left(2^{\prime}\right)} \equiv$ $\kappa^{2} L_{\text {generic }}^{(2)}$ are completely similar to the case of GR. This is also a more familiar manner which has been used by Lovelock 24] for the non-generic case, though he then proceeded from this to derive equation (4).

The "trace" relation, Trace $R_{\text {(generic) } \alpha \beta}^{\left(2^{\prime}\right)}=R_{\text {generic }}^{\left(2^{\prime}\right)}$, holds if and only if, for non zero coefficients,

$$
(D-1) a_{1}+\frac{D}{4} a_{2}+a_{3}=0 \quad \text { for } D \geq 2 .
$$

As it is evident, the explicit appearance of $D$ gives rise to a dimensional dependent version of Duff's trace anomaly relation. Though, one may ar- 
gue that usually the trace anomaly in $2 D$ dimensions is related to $R^{D_{-}}$ like invariants, i.e. the $D$ order Lagrangian terms. Actually, investigation shows 94, 96] that similar constraint relations for the third order Lagrangian terms hold which Weyl invariants in six dimensions [88, 98, do satisfy them.

Again, the constraint (27) gives two degrees of freedom, in $D>4$, to choose a desired combination. Also, only in four dimensions, it is exactly the same as the condition (10), where, due to the extra condition of the Gauss-Bonnet density, leads again in this dimension to the condition (24). Hence, the constraint has been modified for higher dimensions. The reason is obvious, for that one can generally set

$$
\begin{aligned}
& R_{\alpha \beta}^{\prime} \equiv R_{(\text {generic } \alpha \beta}^{(2)}-g_{\alpha \beta} P \\
& R^{\prime} \equiv R_{\text {generic }}^{(2)}-2 P
\end{aligned}
$$

where $P$ can be an arbitrary homogeneous function of any order jet-prolongation of the metric with the HDN two. But, using (6), one gets

$$
\text { Trace } R_{\alpha \beta}^{\prime}=R_{\text {generic }}^{(2)}-\frac{D}{2} P \text {. }
$$

And, in order to be equal to $R^{\prime}, D$ must be four. In the special case of $R_{\text {(generic) } \alpha \beta}^{\left(2^{\prime}\right)}$ and $R_{\text {generic }}^{\left(2^{\prime}\right)}, P$ is obviously proportional to $\square R$. Incidentally, since the term $\square R$ is a complete divergence, it gives no contribution to the variation of the relevant action. Therefore, adding such a term to the Lagrangian still gives the same result, but it can cover the remaining part of the trace anomaly (14).

The non-generic combination of the Lanczos Lagrangian satisfies the above constraint in any dimension. Actually, one can write equation (27) as

$$
D\left(a_{1}+\frac{1}{4} a_{2}\right)+\left(a_{3}-a_{1}\right)=0,
$$

which shows that the only combination that is independent of dimension is the combination of the Lanczos Lagrangian.

The square of the Weyl conformal tensor, equation (22), identically satisfies the condition (27) independent of dimension. Hence, the modified derivation of the "trace" relation yields equation (27) which now does give the Euler density invariant and the Weyl squared in arbitrary dimensions.

To reiterate, if one considers only one of the second order Lagrangians (7) in isolation, constraint (27) will set the third coefficient equal to zero as well. In three dimensions, again because of the extra Gauss-Bonnet relation, the constraint, for the Lagrangian (1), now reads

$$
\frac{8}{3} \alpha+\beta=0
$$


In this case, the particle spectrum in the quantum theory should be rechecked [113]. In two dimensions, the constraint gives

$$
a_{1}+\frac{1}{2} a_{2}+a_{3}=0
$$

where, because of the relation mentioned before, the Lagrangian under consideration actually is $\left(a_{1}+\frac{1}{2} a_{2}+a_{3}\right) R^{2}$. Hence, the above constraint, in two dimensions, again confirms the null result of the previous section. But, the arrangement (25), for the cosmological term, cannot be performed.

Finally, the Euler-Lagrange expression $G_{(\text {generic) } \alpha \beta}^{(2)}$ could partially be disarranged and be written as

$$
\begin{aligned}
& G_{\text {(generic) } \alpha \beta}^{(2)}= \\
& 2\left\{a_{1} R R_{\alpha \beta}-2 a_{3} R_{\alpha \mu} R_{\beta}{ }^{\mu}+a_{3} R_{\alpha \rho \mu \nu} R_{\beta}{ }^{\rho \mu \nu}+\left(a_{2}+2 a_{3}\right) R_{\alpha \mu \beta \nu} R^{\mu \nu}-\left(a_{1}\right.\right. \\
& \left.\left.\quad+\frac{1}{2} a_{2}+a_{3}\right) R_{; \alpha \beta}+\left(\frac{1}{2} a_{2}+2 a_{3}\right) \square R_{\alpha \beta}+a\left[\frac{1}{4}\left(4 a_{1}+a_{2}\right)+a_{3}\right] g_{\alpha \beta} \square R\right\} \\
& -\frac{1}{2} g_{\alpha \beta}\left\{\kappa^{2} L_{\text {generic }}^{(2)}+\left[\left(4 a_{1}+a_{2}\right)(a-1)+4 a a_{3}\right] \square R\right\},
\end{aligned}
$$

where $a$ is a number. In this case, the "trace" requirement holds if and only if

$$
a_{1}[a(D-4)+3]+a_{2}\left[\frac{a}{4}(D-4)+1\right]+a_{3}[a(D-4)+1]=0 .
$$

The most interesting point of the above condition is that, in four dimensions it reduces to condition (10) irrespective of the "disarrangement" factor $a$.

\section{Acknowledgement}

This work has been supported by the research council of the Shahid Beheshti University, Tehran, Iran.

\section{References}

[1] Isham, C J "Quantum gravity" in Proc. $11^{\text {th }}$ General Relativity and Gravitation, Stockholm, 1986, Ed. M A H MacCallum (Cambridge University Press, 1987), pp 99-129; "Structural issues in quantum gravity", $g r-q c / 9510063$

[2] Newman, E T \& Goenner, H "Classical and quantum alternatives to gravitational theories" in Proc. $10^{\text {th }}$ General Relativity and Gravitation, Padua, Italy, 1983, Eds. B Bertotti, F de Felice \& A Pascolini (D Reidel Publishing Company, Holland, 1984), pp 199-211; 
Goenner, H "Alternative theories of gravity" in Proc. $11^{\text {th }}$ General Relativity and Gravitation, Stockholm, 1986, Ed. M A H MacCallum (Cambridge University Press, 1987), pp 262-273;

Francaviglia, M "Alternative gravity theories" in Proc. $12^{\text {th }}$ General Relativity and Gravitation, Boulder, 1989, Eds. N Ashby, D F Bartlett \& W Wyss (Cambridge University Press, 1990), pp 99-104.

[3] Hilbert, D "Die Grundlagen der Physik", I \& II, Nachr. Gesel. Wiss. Göttingen, (1915), 395-407 and (1917), 53-76, respectively. These were consolidated with the same title into: Math. Annalen 92 (1924), 1-32.

[4] Weyl, H "Gravitation und Elektrizität", Preuss. Akad. Wiss. Berlin, Sitz. (1918), 465-480; "Eine neue Erweiterung der Relativitätstheorie", Ann. der Phys. 59 (1919), 101-133; Raum-Zeit-Materie, (SpringerVerlag, Berlin, $1^{\text {st }}$ ed. 1918, $4^{\text {th }}$ ed. 1921). Its English version (of the $4^{\text {th }}$ ed.) is: Space-Time-Matter, translated by: H L Brose (Dover Publications, New York, $1^{\text {st }}$ ed. 1922, reprinted 1950); "Über die physikalischen Grundlagen der erweiterten Relativitätstheorie", Phys. Zeitschr. 22 (1921), 473-480.

[5] Eddington, A, The Mathematical Theory of Relativity, (Cambridge University Press, $1^{\text {st }}$ ed. 1923, $2^{\text {nd }}$ ed. 1924; Chelsee Publishing Co., New York, 1975).

[6] Stelle, K S "Classical gravity with higher derivatives", Gen. Rel. Grav. 9 (1978), 353-371.

[7] Maluf, W "Conformal invariance and torsion in general relativity", Gen. Rel. Grav. 19 (1987), 57-71.

[8] Bach, R "Zur Weylschen Relativitätstheorie und der Weylschen Erweiterung des Krümmungstensorbegriffs", Math. Zeitschr. 9 (1921), 110135 .

[9] Lanczos, C "Elektromagnetismus als natürliche Eigenschaft der Riemanns chen Geometrie", Zeits. Phys. 73 (1931), 147-168; "Electricity and general relativity", Rev. Mod. Phys. 29 (1957), 337-350.

[10] Buchdahl, H A "On the gravitational field equations arising from the square of a Gaussian curvature", Nuovo Cim. 23 (1962), 141-157;

Bicknell, G V "Non-viability of gravitational theory based on a quadratic Lagrangian", J. Phys. A: Math. Nucl. Gen. 7 (1974), 1061-1069.

[11] Clifton, T \& Barrow, J D "The power of general relativity", gr-qc/0509059.

[12] Barrow, J D \& Clifton, T "Exact cosmological solutions of scaleinvariant gravity theories", $g r-q c / 0509085$ 
[13] Buchdahl, H A "Non-linear Lagrangians and cosmological theory", Mon. Not. R. Astr. Soc. 150 (1970), 1-8.

[14] Wheeler, J A, Einstein's Vision, (Springer-Verlag, Berlin, 1968).

[15] Isham, C J "Structural issues in quantum gravity", gr-qc/9510063, (lectures delivered at the GR14 Conference, Florence, August 1995).

[16] Cecotti, S, Ferrara, S, Girardello, L, Porrati, M \& Pasquinucci, A "Matter coupling in higher derivative supergravity", Phys. Rev. D33 (1986), R2504-R2507.

[17] Birrell, N D \& Davies, P C W, Quantum Fields in Curved Space, (Cambridge University Press, 1982);

Buchbinder, I L, Odintsov, S D \& Shapiro, I L, Effective Action in Quantum Gravity, (Institute of Physics Publishing Bristol and Philadelphia, 1992).

[18] Utiyama, R \& DeWitt, B S "Renormalization of a classical gravitational interacting with quantized matter fields", J. Math. Phys. 3 (1962), 608618 ;

Pechlaner, E \& Sexl, R "On quadratic Lagrangians in general relativity", Commun. Math. Phys. 2 (1966), 165-175.

[19] Chern, S-S "A simple intrinsic proof of the Gauss-Bonnet formula for closed Riemannian manifolds", Ann. Math. 45 (1944), 747-752; "On the curvatura integra in a Riemannian manifold", ibid. 46 (1945), 674-684;

Kobayashi, S \& Nomizu, K, Foundations of Differential Geometry, Vol. II, (Wiley Interscience, New York, 1969);

Spivak, M, A Comprehensive Introduction to Differential Geometry, Vol. 5, (Publish or Perlish Inc., Delaware, $2^{\text {nd }}$ ed. 1979).

[20] Stelle, K S "Renormalization of higher derivative quantum gravity", Phys. Rev. D16 (1977), 953-969.

[21] Fradkin, E S \& Tseytalin, A A "Renormalizable asymptotically free quantum theory of gravity", Nucl. Phys. B201 (1982), 469-491.

[22] Candelas, P, Horowitz, G T, Strominger, A \& Witten, E "Vacuum configurations for superstrings", Nucl. Phys. B258 (1985), 46-74;

Metsaev, R R \& Tseytlin, A A "Curvature cubed terms in string theory effective actions", Phys. Lett. B185 (1987), 52-58;

Green, M B, Schwarz, J H \& Witten, E, Superstring Theory, Vols. 1 \& 2, (Cambridge University Press, 1987);

Lust, D \& Theusen, S, Lectures on String Theory, (Springer, Berlin, 1989);

Ketov, S V "The string generated correction to Einstein gravity from 
the sigma model approach", Gen. Rel. Grav. 22 (1990), 193-202;

Polchinski, J, String Theory, (Cambridge University Press, 1998).

[23] Deser, S "Gravity from strings", Phys. Scripta T15 (1987), 138-142.

[24] Lovelock, D "The Einstein tensor and its generalizations", J. Math. Phys. 12 (1971), 498-501; "The four dimensionality of space and the Einstein tensor", ibid. 13 (1972), 874-876.

[25] Briggs, C C "Some possible features of general expressions for Lovelock tensors and for the coefficients of Lovelock Lagrangians up to the $15^{\text {th }}$ order in curvature (and beyond)", $g r-q c / 9808050$.

[26] Wald, R M, General Relativity, (The University of Chicago Press, 1984).

[27] Zwiebach, B "Curvature squared terms and string theories", Phys. Lett. B156 (1985), 315-317;

Zumino, B "Gravity theories in more than four dimensions", Phys. Rep. 137 (1986), 109-114.

[28] Duff, M J, Nilsson, B E W \& Pope, C N "Gauss-Bonnet from KaluzaKlein", Phys. Lett. B173 (1986), 69-72.

[29] Chamseddine, A H "Topological gauge theory of gravity in five and all odd dimensions", Phys. Lett. B233 (1989), 291-294;

Müller-Hoissen, F "From Chern-Simons to Gauss-Bonnet", Nucl. Phys. B346 (1990), 235-252.

[30] Allemandi, G, Francaviglia, M \& Raiteri, M "Charges and energy in Chern-Simons theories and Lovelock gravity", Class. Quantum Grav. 20 (2003), 5103-5120.

[31] Nojiri, S \& Odintsov, S D "Where new gravitational physics comes from M-theory?", Phys. Lett. B576 (2003), 5-11.

[32] Simon, J Z "Higher derivative Lagrangians, non-locality, problems, and solutions", Phys. Rev. D41 (1990), 3720-3733.

[33] Madore, J "Cosmological applications of the Lanczos Lagrangian", Class. Quantum Grav. 3 (1986), 361-371;

Deruelle, N \& Madore, J "Kaluza-Klein cosmology with the Lovelock Lagrangian" in Origin and Early History of the Universe, Proc. $26^{\text {th }}$ Liège Int. Astrophysical Colloquium, Belgium, July 1986, (Cointe-Ougree, Belgium, 1987), pp 277-283;

Mena Marugán, G A "Classical and quantum Lovelock cosmology", Phys. Rev. D42 (1990), 2607-2620; "Lovelock gravity and classical wormholes", Class. Quantum Grav. 8 (1991), 935-946;

Poisson, E "Quadratic gravity and the black hole singularity", Phys. 
Rev. D43 (1991), 3923-3928;

Cotsakis, S, Cosmological Models in Higher Order Gravity, (Ph.D. Thesis, Sussex University, 1990);

Fariña-Busto, L, Non-Linear Gravitational Lagrangians in Cosmology, (Ph.D. Thesis, Queen Mary \& Westfield College, University of London, 1990).

[34] Carloni, S, Dunsby, P K S, Capozziello, S \& Troisi, A "Cosmological dynamics of $R^{n}$ gravity", Class. Quantum Grav. 22 (2005), 4839-4868.

[35] Berkin, A L \& Maeda, K "Effects of $R^{3}$ and $R \square R$ terms on $R^{2}$ inflation", Phys. Lett. B245 (1990), 348-354.

[36] Schmidt, H-J "Variational derivatives of arbitrarily high order and multi-inflation cosmological models", Class. Quantum Grav. 7 (1990), 1023-1031;

Berkin, A L \& Maeda, K "Inflation in generalized Einstein theories", Phys. Rev. D44 (1991), 1691-1704.

[37] Gottlöber, S, Müller, V \& Schmidt, H-J "Generalized inflation from $R^{3}$ and $R \square R$ terms", Astron. Nachr. 312 (1991), 291-297.

[38] Dehghani, M H "Magnetic branes in Gauss-Bonnet gravity", Phys. Rev. D69 (2004), 064024;

Meng, X-H, \& Wang, P "Inflationary attractor in Gauss-Bonnet brane cosmology", Class. Quantum Grav. 21 (2004), 2527-2536;

Allemandi, G, Borowiec, A \& Francaviglia, M "Accelerated cosmological models in Ricci squared gravity", Phys. Rev. D70 (2004), 103503.

[39] Dehghani, M H "Accelerated expansion of the universe in GaussBonnet gravity", Phys. Rev. D70 (2004), 064009.

[40] Riess, A G, et al "Observational evidence from supernovae for an accelerating universe and a cosmological constant", Astro. J. 116 (1998), 1009-1038;

Perlmutter, S, et al "Measurements of omega and lambda from 42 highredshift supernovae", Astrophys. J. 517 (1999), 565-586;

Tonry, J L, et al "Cosmological results from high-z supernovae", Astrophys. J. 594 (2003), 1-24.

[41] Lee, A T, et al "A high spatial resolution analysis of the MAXIMA1 cosmic microwave background anisotropy data", Astrophys. J. 561 (2001), L1-L6;

Netterfield, C B, et al "A measurement by BOOMERANG of multiple peaks in the angular power spectrum of the cosmic microwave background", Astrophys. J. 571 (2002), 604-614; 
Halverson, N W, et al "DASI first results: a measurement of the cosmic microwave background angular power spectrum", Astrophys. J. 568 (2002), 38-45;

Spergel, D N, et al "First year Wilkinson Microwave Anisotropy Probe (WMAP) observations: determination of cosmological parameters", Astrophys. J. Suppl. 148 (2003), 175-225.

[42] Carroll, S M, Duvvuri, V, Trodden, M \& Turner, M S "Is cosmic speedup due to new gravitational physics?", Phys. Rev. D70 (2004), 043528; Capozziello, S, Cardone, V F, Carloni, S \& Troisi, A "Curvature quintessence matched with observational data", Int. J. Mod. Phys. D12 (2003), 1969-1982; "Higher order curvature theories of gravity matched with observations: a bridge between dark energy and dark matter problems", astro-ph/0411114.

[43] Nojiri, S \& Odintsov, S D "Modified gravity with $\ln R$ terms and cosmic acceleration", Gen. Rel. Grav. 36 (2004), 1765-1780.

[44] Nojiri, S \& Odintsov, S D "Modified gravity with negative and positive powers of the curvature: unification of the inflation and of the cosmic acceleration", Phys. Rev. D68 (2003), 123512.

[45] Chiba, T “ $\frac{1}{R}$ gravity and scalar-tensor gravity", Phys. Lett. B575 (2003), 1-3.

[46] Soussa, M E \& Woodard, R P "The force of gravity from a Lagrangian containing inverse powers of the Ricci scalar", Gen. Rel. Grav. 36 (2004), 855-862.

[47] Dolgov, A D \& Kawasaki, M "Can modified gravity explain accelerated cosmic expansion?", Phys. Lett. B573 (2003), 1-4.

[48] Nojiri, S \& Odintsov, S D "The minimal curvature of the universe in modified gravity and conformal anomaly resolution of the instabilities", Mod. Phys. Lett. A19 (2004), 627-638.

[49] Madsen, M S \& Barrow, J D "De Sitter ground states and boundary terms in generalized gravity", Nucl. Phys. B323 (1989), 242-252.

[50] Mardones, A \& Zanelli, J "Lovelock-Cartan theory of gravity", Class. Quantum Grav. 8 (1991), 1545-1558.

[51] Palatini, A "Deduzione invariantiva delle equazioni gravitazionali dal principio di Hamilton", Rend. Circ. Mat. Palermo 43 (1919), 203-212; Stephenson, G "Variational principles \& gauge theories of gravitation", J. Phys. A10 (1977), 181-184. 
[52] Buchdahl, H A "Quadratic Lagrangians and Palatini's device", J. Phys. A12 (1979), 1229-1234.

[53] Shahid-Saless, B "Palatini variation of curvature squared action and gravitational collapse", J. Math. Phys. 32 (1991), 694-697.

[54] Meng, X-H, \& Wang, $\mathrm{P}$ " $R^{2}$ corrections to the cosmological dynamics of inflation in the Palatini formulation", Class. Quantum Grav. 21 (2004), 2029-2036; "Palatini formulation of the $R^{-1}$ modified gravity with an additionally squared scalar curvature term", ibid. 22 (2005), 23-32;

Sotiriou, T P "Unification of inflation and cosmic acceleration in the Palatini formalism", gr-qc/0509029.

[55] Vollick, D N “ $\frac{1}{R}$ curvature corrections as the source of the cosmological acceleration", Phys. Rev. D68 (2003), 063510;

Meng, X-H, \& Wang, $\mathrm{P}$ "Modified Friedmann equations in $R^{-1}$-modified gravity", Class. Quantum Grav. 20 (2003), 4949-4962; "Cosmological evolution in $\frac{1}{R}$ gravity theory", ibid. 21 (2004), 951-960;

Capozziello, S, Cardone, V F \& Francaviglia, M " $f(R)$ theories of gravity in Palatini matched with observations", astro-ph/0410135.

[56] Olmo, G J \& Komp, W "Non-linear gravity theories in the metric and Palatini formalisms", $g r-q c / 0403092$.

[57] Flanagan, E E "Palatini form of $\frac{1}{R}$ gravity", Phys. Rev. Lett. 92 (2004), 071101; "Higher order gravity theories and scalar tensor theories", Class. Quantum Grav. 21 (2004), 417-426;

Meng, X-H, \& Wang, P "Palatini formulation of modified gravity wih squared scalar curvature", astro-ph/0308284: "Palatini formulation of modified gravity with $\ln R$ terms", Phys. Lett. B584 (2004), 1-7.

[58] Vollick, D N "On the viability of the Palatini form of $\frac{1}{R}$ gravity", Class. Quantum Grav. 21 (2004), 3813-3816.

[59] Kerner, R "Cosmology without singularity and nonlinear gravitational Lagrangians", Gen. Rel. Grav. 14 (1982), 453-469;

Madore, $\mathrm{J}$ "On the nature of the initial singularity in a Lanczos cosmological model", Phys. Lett. 111A (1985), 283-284;

Horowitz, G T \& Steif, A R "Spacetime singularities in string theory", Phys. Rev. Lett. 64 (1990), 260-263.

[60] Barrow, J D \& Ottewill, A C "The stability of general relativistic cosmological theory", J. Phys. A16 (1983), 2757-2776;

Müller, V, Schmidt, H-J \& Starobinsky A A "The stability of the de Sitter space-time in fourth order gravity", Phys. Lett. B202 (1988), 198-200. 
[61] Faraoni, V "Modified gravity and the stability of de Sitter space", Phys. Rev. D72 (2005), 061501.

[62] Teyssandier, $\mathrm{P}$ "Linearized $R+R^{2}$ gravity: a new gauge and new solutions", Class. Quantum Grav. 6 (1989), 219-229.

[63] Quant, I \& Schmidt, H-J "The Newtonian limit of fourth and higher order gravity", Astron. Nachr. 312 (1991), 97-102.

[64] Dick, $\mathrm{R}$ "On the Newtonian limit in gravity models with inverse power of R", Gen. Rel. Grav. 36 (2004), 217-224;

Meng, X-H, \& Wang, P "Gravitational potential in Palatini formulation of modified gravity", Gen. Rel. Grav. 36 (2004), 1947-1954;

Domínguez, A E \& Barraco, D E "Newtonian limit of the singular $f(R)$ gravity in the Palatini formalism", Phys. Rev. D70 (2004), 043505;

Capozziello, S "Newtonian limit of extended theories of gravity", gr-qc/0412088

Olmo, G J "The gravity Lagrangian according to solar system experiments", $g r-q c / 0505101$ "Post-Newtonian constraints on $f(R)$ cosmologies in metric formalism", $g r-q c / 0505135$ "Post-Newtonian constraints on $f(R)$ cosmologies in Palatini formalism", $g r-q c / 0505136$.

Allemandi, G, Francaviglia, M, Ruggiero, M L \& Tartaglia, A "Post-Newtonian parameters from alternative theories of gravity", gr-qc/0506123

Sotiriou, T P "The nearly Newtonian regime in non-linear theories of gravity", $g r-q c / 0507027$

[65] Cembranos, J A R "The Newtonian limit at intermediate energies", gr-qc/0507039

[66] Capozziello, S \& Troisi A "PPN-limit of fourth order gravity inspired by scalar-tensor gravity", gr-qc/0507545.

[67] Teyssandier, $\mathrm{P} \&$ Tourrenc, $\mathrm{Ph}$ "The Cauchy problem for the $R+R^{2}$ theories of gravity without torsion", J. Math. Phys. 24 (1983), 27932799 .

[68] Strominger, A "Positive energy theorem for $R+R^{2}$ gravity", Phys. Rev. D30 (1984), 2257-2259;

Maluf, W "Energy spectrum of quadratic theories of gravitation", Class. Quantum Grav. 6 (1989), 1189-1195; "Positivity of energy of $R+R^{2}$ theories of gravitation", ibid., L151-L154.

[69] Magnano, G \& Sokolowski, L M "On physical equivalence between nonlinear gravity theories and a general relativistic self-gravitating scalar field", Phys. Rev. D50 (1994), 5039-5059. 
[70] Magnano, G, Ferraris, M \& Francaviglia, M "Non-linear gravitational Lagrangians", Gen. Rel. Grav. 19 (1987), 465-479; "Legendre transformation and dynamical structure of higher derivative gravity", Class. Quantum Grav. 7 (1990), 557-570;

Sirousse Zia, H "Singularity theorems and the [general relativity + additional matter fields] formulation of metric theories of gravitation", Gen. Rel. Grav. 26 (1994), 587-597.

[71] Brans, C H "Non-linear Lagrangians and the significance of the metric", Class. Quantum Grav. 5 (1988), L197-L199.

[72] Ferraris, M, Francaviglia, M \& Magnano, G "Do non-linear metric theories of gravitation really exist?", Class. Quantum Grav. 5 (1988), L95L99; "Remarks on the physical metric in non-linear theories of gravitation", ibid. 7 (1990), 261-263;

Sokolowski, L M "Physical versions of non-linear gravity theories and positivity of energy", Class. Quantum Grav. 6 (1989), 2045-2050.

[73] Pascual-Sánchez, J-F "Variational principles and quantum gravity" in Recent Developments in Gravitation, Proc. Relativity Meeting, Barcelona, Spain, Sept. 1989, Eds. E Verdaguer, J Garriga \& J Céspedes (World Scientific, Singapore, 1990), pp 397-404.

[74] Barrow, J D \& Cotsakis, S "Inflation and the conformal structure of higher order gravity theories", Phys. Lett. B214 (1988), 515-518;

Maeda, K "Towards the Einstein-Hilbert action via conformal transformation", Phys. Rev. D39 (1989), 3159-3162;

Wands, D "Extended gravity theories and the Einstein-Hilbert action", Class. Quantum Grav. 11 (1994), 269-280;

Hindawi, A, Ovrut, B A \& Waldram, D "Non-trivial vacua in higher derivative gravitation", Phys. Rev. D53 (1996), 5597-5608.

[75] Gottlöber, S, Schmidt, H-J \& Starobinsky, A A "Sixth-order gravity and conformal transformations", Class. Quantum Grav. 7 (1990), 893900.

[76] Boulware, D G \& Deser, S "String-generated gravity models", Phys. Rev. Lett. 55 (1985), 2656;

Cai, R G \& Soh, K S "Topological black holes in the dimensionally continued gravity", Phys. Rev. D59 (1999), 044013;

Nojiri, S, Odintsov, S D \& Ogushi, S "Cosmological and black hole brane-world universe in higher derivative gravity", Phys. Rev. D65 (2002), 023521;

Cvetic, M, Nojiri, S \& Odintsov, S D "Black hole thermodynamics and negative entropy in de Sitter and anti-de Sitter Einstein-Gauss-Bonnet gravity", Nucl. Phys. B628 (2002), 295-330; 
Cai, R G "Gauss-Bonnet black holes in AdS spaces", Phys. Rev. D65 (2002), 084014;

Cho, Y M \& Neupane, I P "Anti-de Sitter black holes, thermal phase transition and holography in higher curvature gravity", Phys. Rev. D66 (2002), 024044.

[77] Maartens, R \& Taylor, D R "Fluid dynamics in higher order gravity", Gen. Rel. Grav. 26 (1994), 599-613;

Rippl, S, van Elst, H, Tavakol, R \& Taylor, D "Kinematics and dynamics of $f(R)$ theories of gravity", Gen. Rel. Grav. 28 (1996), 193-205;

Brevik, I, Nojiri, S, Odintsov, S D \& Vanzo, L "Entropy and universality of Cardy-Verlinde formula in dark energy universe", Phys. Rev. D70 (2004), 043520.

[78] Buchdahl, H A "Über die Variationsableitung von fundamentalinvarianten beliebig hoher Ordnung", Acta Math. 85 (1951), 63-72.

[79] Amendola, L, Mayer, A B, Capozziello, S, Gottlöber, S, Müller, V, Occhionero, F \& Schmidt, H-J "Generalized sixth order gravity and inflation", Class. Quantum Grav. 10 (1993), L43-L47.

[80] Stephenson, G "Variational principles for the gravitational field", Lett. Nuovo Cim. 1 (1969), 97-99.

[81] Roxburgh, I W "Non-linear Lagrangian theories of gravity", Gen. Rel. Grav. 8 (1977), 219-225;

Coley, A A "Homothetic vectors and higher order Lagrangian theories of gravity", Class. Quantum Grav. 6 (1989), 1213-1218.

[82] Farhoudi, M "Lovelock tensor as generalized Einstein tensor", gr-qc/9510060.

[83] Farhoudi, M "Classical trace anomaly", Int. J. Mod. Phys. D 14 (2005), 1233-1250.

[84] Duff, M J "Twenty years of the Weyl anomaly", Class. Quantum Grav. 11 (1994), 1387-1403.

[85] Asorey, M, Gorbar, E V \& Shapiro, I L "Universality and ambiguities of the conformal anomaly", Class. Quantum Grav. 21 (2003), 163-178.

[86] Duff, M J "Observations on conformal anomalies", Nucl. Phys. B125 (1977), 334-348.

[87] Bonora, L, Cotta-Ramusino, P \& Reina, C "Conformal anomaly and cohomology", Phys. Lett. 126B (1983), 305-308.

[88] Bonora, L, Pasti, P \& Bregola, M "Weyl cocycles", Class. Quantum Grav. 3 (1986), 635-649. 
[89] Nojiri, S, Odintsov, S D \& Ogushi, S "Holographic renormalization group and conformal anomaly for $\mathrm{AdS}_{9} / \mathrm{CFT}_{8}$ correspondence", Phys. Lett. B500 (2001), 199-208.

[90] Deser, S, Duff, M J \& Isham, C J "Non-local conformal anomalies", Nucl. Phys. B111 (1976), 45-55;

Christensen, S M "Regularization, renormalization, and covariant geodesic point separation", Phys. Rev. D17 (1978), 946-963.

[91] Avramidi, I G "New algebraic methods for calculating the heat kernel and effective action in quantum gravity and gauge theories" in Heat Kernel Techniques and Quantum Gravity, Discourses in Mathematics and its Applications, Ed. S A Fulling, (Department of Mathematics, Texas A\&M University, 1995), pp 115-140, gr-qc/9408028.

[92] Fulton, T, Rohrlich, F \& Witten, L "Conformal invariance in physics", Rev. Mod. Phys. 34 (1962), 442-457.

[93] Müller-Hoissen, F "Spontaneous compactification with quadratic and cubic curvature term", Phys. Lett. 163B (1985), 106-110.

[94] Farhoudi, M Non-linear Lagrangian Theories of Gravitation, (Ph.D. Thesis, Queen Mary \& Westfield College, University of London, 1995).

[95] Wheeler, J T "Symmetric solutions to the Gauss-Bonnet extended Einstein equations", Nucl. Phys. B268 (1986), 737-746.

[96] Farhoudi, M "New derivation of Weyl invariants in six dimensions", work in progress.

[97] Deruelle, N \& Fariña-Busto, L "The Lovelock gravitational field equations in cosmology", Phys. Rev. D41 (1990), 3696-3708.

[98] Deser, S \& Schwimmer, A "Geometric classification of conformal anomalies in arbitrary dimensions", Phys. Lett. B309 (1993), 279-284.

[99] Hamber, H W \& Williams, R M "Two dimensional simplical quantum gravity", Nucl. Phys. B267 (1986), 482-496;

Katanayev, M O \& Volovich, I V "Two dimensional gravity with dynamical torsion and string", Ann. Phys. 197 (1990), 1-32;

Yoneya, T "Higher derivative quantum gravity in two dimensions", Phys. Lett. 149B (1984), 111-116.

[100] Brown, J D, Lower Dimensional Gravity, (World Scientific, Singapore, 1988). 
[101] Stachel, J "Einstein's struggle with general covariance, 1912-1915" presented at General Relativity and Gravitation $9^{\text {th }}, 1980$ at Jena, Germany; reprinted as "Einstein's search for general covariance, 1912-1915" in Proc. Einstein and the History of General Relativity, Osgood Hill Conference, Massachusetts, May 1986, Eds. D Howard \& J Stachel (The Center for Einstein Studies, Boston Univ., 1989), pp 63-100.

[102] Einstein, A, Wrote to: P Ehrenfest, on $26^{\text {th }}$ December, 1915, EA 9363 ;

Wrote to: M Besso, on $3^{\text {rd }}$ January, 1916 in Albert Einstein, Michele Besso, Correspondence, 1903-1955, Ed. P Speziali (Hermann, Paris, 1972), pp 63-64.

[103] Einstein, A "Relativity and the problem of space" (1952), Appendix 5 in Relativity, the Special and the General Theory: A Popular Exposition, translated by: R W Lawson (Methuen, London, $15^{\text {th }}$ edition 1954), pp 135-157.

[104] Adler, I, A New Look at Geometry, (John Day Com., New York, 1966).

[105] Mashhoon, B, Liu, H \& Wesson, P S "Space-time-matter" in Proc. $7^{\text {th }}$ Marcel Grossmann Meeting, Stanford, 1994, pp 333-335.

[106] Graves, J C, The Conceptual Foundation of Contemporary Relativity Theory, (MIT Press, 1971).

[107] Ryan, Jr., M P \& Shepley, L C, Homogeneous Relativistic Cosmologies, (Princeton Univ. Press, 1975).

[108] Schwarzchild, k "Über das gravitationsfeld eines massenpunktes nach der Einsteinschen theorie", Sitzber. Preuss. Akad. Wiss. Berlin (1916), pp 189-196;

Taub, A H "Empty space-times admitting a three parameter group of motions", Ann. Math. 53 (1951), 472-490;

Newman, E T, Tamburino, L \& Unti, T J "Empty-space generalization of the Schwarzchild metric", J. Math. Phys. 4 (1963), 915-923.

[109] Abbassi, A M Revisiting the Mach's and Correspondence Principle in General Relativity and Concept of Inertia, (Ph.D. Thesis, Tarbiat Modarres University, Tehran, Iran, 2001).

[110] Salam, A "Gauge unification of fundamental forces", Rev. Mod. Phys. 52 (1980), 525-538.

[111] Romero, C, Tavakol, R \& Zalaletdinov, R "The embedding of general relativity in five dimensions", Gen. Rel. Grav. 28 (1996), 365-376. 
[112] Wesson, P S, Space-Time-Matter, Modern Kaluza-Klein Theory, (World Scientific, Singapore, 1999);

Wesson, P S "In defence of Campbell's theorem as a frame for new physics", gr-qc/0507107.

[113] Farhoudi, M, work in progress. 